\title{
CONCURRENT TERNARY GALOIS-BASED COMPUTATION USING NANO-APEX MULTIPLEXING Nibs OF REgular THREE-DimensionaL NETWORKS, PART II: ForMalistic ARCHITECTURE REALIZATION
}

\author{
Anas N. Al-Rabadi \\ Department of Computer Engineering, The University of Jordan, Amman - Jordan \& \\ Department of Renewable Energy Engineering, Isra University - Jordan
}

\begin{abstract}
Novel realizations of concurrent computations utilizing three-dimensional lattice networks and their corresponding carbon-based field emission controlled switching is introduced in this article. The formalistic ternary nano-based implementation utilizes recent findings in field emission and nano applications which include carbon-based nanotubes and nanotips for three-valued lattice computing via field-emission methods. The presented work implements multi-valued Galois functions by utilizing concurrent nano-based lattice systems, which use two-to-one controlled switching via carbon-based field emission devices by using nano-apex carbon fibers and carbon nanotubes that were presented in the first part of the article. The introduced computational extension utilizing many-to-one carbon field-emission devices will be further utilized in implementing congestion-free architectures within the third part of the article. The emerging nano-based technologies form important directions in low-power compact-size regular lattice realizations, in which carbon-based devices switch less-costly and more-reliably using much less power than silicon-based devices. Applications include low-power design of VLSI circuits for signal processing and control of autonomous robots.
\end{abstract}

\section{KEYWORDS}

Carbon nano-apex emission, Concurrent computing, Formal symmetrization, Lattice networks, Regularity.

\section{INTRODUCTION}

In general VLSI system design, regular interconnects usually lead to cheap implementations and high densities, where higher density implies both higher performance and lower overhead for support components. Thus, regular circuit topologies that involve the realization of functions in three-dimensions can be very important, as it shows that the best way is to synthesize functions in three-dimensions where all regular local interconnects are of the same length and global interconnects are only inputs on parallel oblique planes [2]-[4].

The class of regular lattice networks [2]-[4] is an important class of regular circuits that generalize the ideas from the well-known Akers arrays [1], spectral transform decision trees and decision diagrams [16], [36]-[37], and symmetric networks [31]. Due to high regularity, lattice networks are useful in many applications including fault-related issues such as testing, localization and self-repair. Since more power consumption occurs whenever more global interconnects are used instead of local interconnects in circuit design [35], lattice networks offer a good solution for the problem of the increase in using global interconnects since lattice networks use internally only local interconnects [2]. 
The method of field electron emission is performed through the emission of electrons from the surface of a cathode under the influence of the applied electric field which is strongly dependent upon the work function of the emitting material [9]-[11], [13]-[15], [18], [24]-[26], [28], where the general form of the governing Fowler-Nordheim equation [25] was produced [24]. In the second part of the article, the utilization of carbon field emission - based devices that implement one fundamental building block in modern logic synthesis known as the controlled switch [33] is introduced, and the use of the presented carbon field emission-based devices in many-valued computations is also shown for the important case of ternary Galois logic. Carbon field emission can be obtained using carbon nanotubes (CNTs) within nanotechnology with wide variety of existing and potential applications [5]-[8], [12], [17], [19]-[23], [29], [32], [34], [38]-[41] and also using carbon nano-apex tips. Figure 1 illustrates the layout of the introduced design method of carbon field emission - based system that is used in this article.

\begin{tabular}{|c|}
\hline Three-Dimensional Formalistic Lattice Realizations \\
\hline Field Emission-Based Circuits \\
\hline Carbon-Based Field Emission Devices \\
\hline Field-Emission Physics \\
\hline Galois Algebra \\
\hline
\end{tabular}

Figure 1. The implemented hierarchical realization of concurrent nano-based lattice networks.

The research findings and implementations in this article are new and original, and are performed for the first time to implement ternary Galois functions using concurrent nano three-dimensional lattice systems that utilize carbon-based field emission devices which are based on field-emission from nano-apex carbon fibers and nanotubes.

The remainder of this article is organized as follows: Fundamentals of ternary Shannon and Davio expansions is presented in Section 2. Basics of formal synthesis of three-dimensional Shannon and Davio lattice networks is presented in Section 3. The utilization of the carbon field emission - based devices in controlled switching and within multi-valued computations is presented in Section 4. The implementation of controlled switching that use carbon field emission - based devices within three-dimensional lattice networks is introduced in Section 5. Conclusions are presented in Section 6.

\section{FUndamental Ternary ShannON ANd Davio ExPANSIONS}

As an important algebraic system, Galois field has been extensively used in several engineering applications including circuit design and testing [2], [30]. Third-radix of Galois field addition and multiplication operations are defined as shown in Tables 1(a) and 1(b), respectively.

Table 1. Galois operations: (a) GF(3) addition, and (b) GF(3) multiplication.

(a)

\begin{tabular}{|c|c|c|c|}
\hline+ & $\mathbf{0}$ & $\mathbf{1}$ & $\mathbf{2}$ \\
\hline $\mathbf{0}$ & 0 & 1 & 2 \\
\hline $\mathbf{1}$ & 1 & 2 & 0 \\
\hline $\mathbf{2}$ & 2 & 0 & 1 \\
\hline
\end{tabular}

(b)

\begin{tabular}{|l|l|l|l|}
\hline $\boldsymbol{*}$ & $\mathbf{0}$ & $\mathbf{1}$ & $\mathbf{2}$ \\
\hline $\mathbf{0}$ & 0 & 0 & 0 \\
\hline $\mathbf{1}$ & 0 & 1 & 2 \\
\hline $\mathbf{2}$ & 0 & 2 & 1 \\
\hline
\end{tabular}


A literal is a function of one variable. The 1-Reduced Post literal (1-RPL) [2], [30], [36] is defined as:

${ }^{i} x=1$ iff $x=i$ else ${ }^{i} x=0$

For example $\left\{{ }^{0} x,{ }^{1} x,{ }^{2} x\right\}$ are the zero, first and second polarities of the 1-RPL, respectively. Also, the ternary shifts of variable $x$ are defined such as $x$ with no shift, $x^{\prime}$ with one shift, and $x^{\prime \prime}$ with two shifts (i.e., $x=x+0, x^{\prime}=x+1$, and $x^{\prime \prime}=x+2$, respectively), where $x$ can take any value in the set $\{0,1,2\}$.

The fundamental Shannon decomposition over GF(3) for a ternary function with a single variable has the following form [2], [27]:

$f={ }^{0} x f_{0}+{ }^{1} x f_{1}+{ }^{2} x f_{2}$

Where $f_{0}$ is cofactor of $f$ with respect to variable $x=0, f_{1}$ is cofactor of $f$ with respect to variable $x$ $=1$, and $f_{2}$ is cofactor of $f$ with respect to variable $x=2$.

Using the addition and multiplication over $\mathrm{GF}(3)$ and the axioms of $\mathrm{GF}(3)$, it can be shown that the ternary 1-RPLs, which are defined in Equation (1), are related to the powers of shifts of variables over $\mathrm{GF}(3)$ as follows [2]:

$$
\begin{aligned}
& { }^{0} x=2(x)^{2}+1 \\
& { }^{0} x=2\left(x^{\prime}\right)^{2}+2\left(x^{\prime}\right) \\
& { }^{0} x=2\left(x^{\prime \prime}\right)^{2}+x^{\prime \prime} \\
& { }^{1} x=2(x)^{2}+2(x) \\
& { }^{1} x=2\left(x^{\prime}\right)^{2}+x^{\prime} \\
& { }^{1} x=2\left(x^{\prime \prime}\right)^{2}+1 \\
& { }^{2} x=2(x)^{2}+x \\
& { }^{2} x=2\left(x^{\prime}\right)^{2}+1 \\
& { }^{2} x=2\left(x^{\prime \prime}\right)^{2}+2\left(x^{\prime \prime}\right)
\end{aligned}
$$

After the substitution of Equations (3) through (11) in Equation (2), and after the minimization of the terms according to the axioms of Galois field, the following Equations are obtained:

$$
\begin{gathered}
f=1 \cdot f_{0}+x \cdot\left(2 f_{1}+f_{2}\right)+2(x)^{2}\left(f_{0}+f_{1}+f_{2}\right) \\
f=1 \cdot f_{2}+x^{\prime} \cdot\left(2 f_{0}+f_{1}\right)+2\left(x^{\prime}\right)^{2}\left(f_{0}+f_{1}+f_{2}\right) \\
f=1 \cdot f_{1}+x^{\prime \prime} \cdot\left(2 f_{2}+f_{0}\right)+2\left(x^{\prime \prime}\right)^{2}\left(f_{0}+f_{1}+f_{2}\right)
\end{gathered}
$$

Equations (2) and (12)-(14) are the ternary Shannon and Davio decompositions for single variable, respectively. These Equations can be rewritten in the following matrix-based forms, respectively:

$$
f=\vec{B}_{S}[\mathrm{~S}] \vec{F}=\left[\begin{array}{lll}
{ }^{0} x & { }^{1} x & 2 \\
2 & {[}
\end{array}\right]\left[\begin{array}{lll}
1 & 0 & 0 \\
0 & 1 & 0 \\
0 & 0 & 1
\end{array}\right]\left[\begin{array}{l}
f_{0} \\
f_{1} \\
f_{2}
\end{array}\right]
$$




$$
\begin{aligned}
& f=\vec{B}_{D 0}\left[\mathrm{D}_{0}\right] \vec{F}=\left[\begin{array}{lll}
1 & x & x^{2}
\end{array}\right]\left[\begin{array}{lll}
1 & 0 & 0 \\
0 & 2 & 1 \\
2 & 2 & 2
\end{array}\right]\left[\begin{array}{l}
f_{0} \\
f_{1} \\
f_{2}
\end{array}\right] \\
& f=\vec{B}_{D 1}\left[\mathrm{D}_{1}\right] \vec{F}=\left[\begin{array}{lll}
1 & x & \left(x^{\prime}\right)^{2}
\end{array}\right]\left[\begin{array}{lll}
0 & 0 & 1 \\
2 & 1 & 0 \\
2 & 2 & 2
\end{array}\right]\left[\begin{array}{l}
f_{0} \\
f_{1} \\
f_{2}
\end{array}\right] \\
& f=\vec{B}_{D 2}\left[\mathrm{D}_{2}\right] \vec{F}=\left[\begin{array}{lll}
1 & x^{\prime \prime} & \left(x^{\prime \prime}\right)^{2}
\end{array}\right]\left[\begin{array}{lll}
0 & 1 & 0 \\
1 & 0 & 2 \\
2 & 2 & 2
\end{array}\right]\left[\begin{array}{l}
f_{0} \\
f_{1} \\
f_{2}
\end{array}\right]
\end{aligned}
$$

Where $\vec{B}_{S}$ and [S] are Shannon basis vector and Shannon spectral transform matrix, $\vec{B}_{D 0}$ and $\left[\mathrm{D}_{0}\right]$ are Davio basis vector and Davio $_{0}$ spectral transform matrix, $\vec{B}_{D 1}$ and $\left[\mathrm{D}_{1}\right]$ are Davio basis vector and Davio 1 spectral transform matrix, $\vec{B}_{D 2}$ and $\left[\mathrm{D}_{2}\right]$ are Davio 2 basis vector and $\mathrm{Davio}_{2}$ spectral transform matrix, and $\vec{F}$ is the truth vector of function $f$.

\section{Formal Synthesis of Three-Dimensional Shannon AND Davio LATTICE NETWORKS}

This section introduces important formal methods for the synthesis of three-dimensional lattice networks that were previously presented in the first part of the article. In general, as a convention, let us denote the nodes in the three-dimensional lattice network by their corresponding tuple $\{x$, $y, \mathrm{z}\}$ within the utilized reference of geometrical three-dimensional Cartesian coordinate system. Also, as a convention within three-dimensional lattice networks, let us denote the edge between two neighboring nodes $\left\{x_{1}, y_{1}, z_{1}\right\}$ and $\left\{x_{2}, y_{2}, z_{2}\right\}$ by the notation $\left\{x_{1}, y_{1}, z_{1}\right\}-\left\{x_{2}, y_{2}, z_{2}\right\}$.

As was presented in the first part of the article, and as will be discussed later in this part of the article, a ternary non-symmetric function is a function that cannot be realized in a threedimensional lattice network without repeating variables [2]. To perform the process of the repetition of variables for an arbitrary non-symmetric ternary function, joining operators are needed to achieve the following objectives of (1) joining the corresponding nodes in threedimensional space and (2) producing the corresponding correction functions in order to preserve the output functionality of the three-dimensional lattice network. In order to obtain the needed joining requirement, Fig. 2 presents such joining operation for the presented three-dimensional lattice networks [2]. 


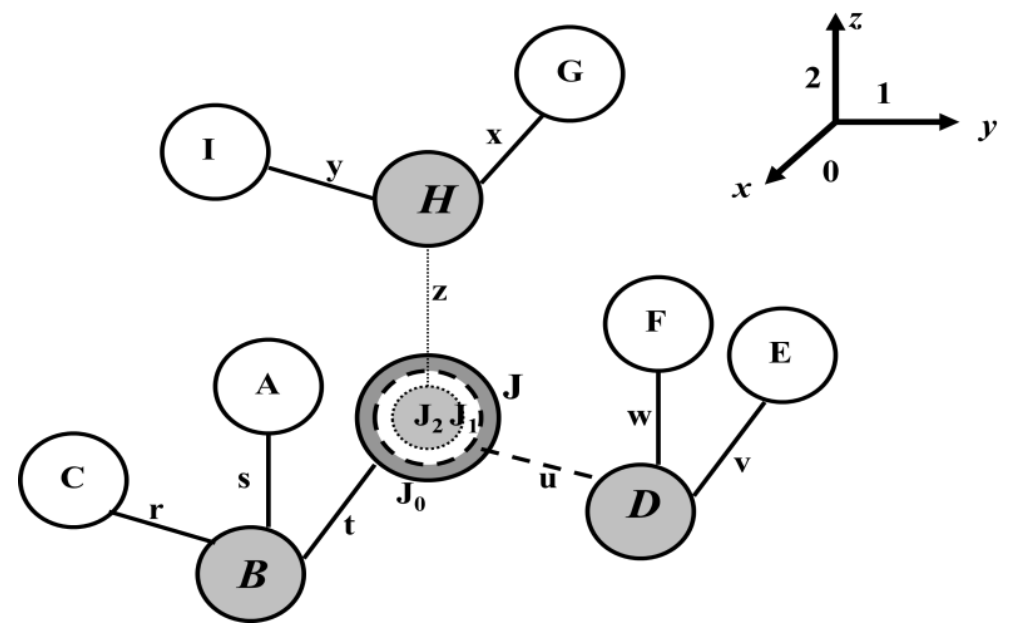

Figure 2. Three joining nodes for realizing a three-dimensional lattice network.

In Fig. 2, three nodes $\{B, D, H\}$ are joining (i.e., super-imposing) their nodes $\left\{\mathrm{J}_{0}, \mathrm{~J}_{1}, \mathrm{~J}_{2}\right\}$ to form the super-imposed node $\{\mathrm{J}\}$. The set of nodes $\left\{\mathrm{C}, \mathrm{A}, \mathrm{J}_{0}\right\}$ are the cofactors of the node $\mathrm{B}$, the set of nodes $\left\{\mathrm{E}, \mathrm{F}, \mathrm{J}_{1}\right\}$ are the cofactors of the node $\mathrm{D}$, and the set of nodes $\left\{\mathrm{G}, \mathrm{I}, \mathrm{J}_{2}\right\}$ are the cofactors of the node H. The geometrical distribution of the nodes and edges in Fig. 2 is shown in Table 2.

Table 2. Three-dimensional placement of nodes and edges from Fig. 2.

\begin{tabular}{|l|l|l|}
\hline Axis & Nodes & Edges \\
\hline$x$-axis & $\left\{\mathrm{J}_{0}, \mathrm{E}, \mathrm{I}\right\}$ & $\{\mathrm{t}, \mathrm{v}, \mathrm{y}\}$ \\
\hline$y$-axis & $\left\{\mathrm{C}, \mathrm{J}_{1}, \mathrm{G}\right\}$ & $\{\mathrm{r}, \mathrm{u}, \mathrm{x}\}$ \\
\hline$z$-axis & $\left\{\mathrm{A}, \mathrm{F}, \mathrm{J}_{2}\right\}$ & $\{\mathrm{s}, \mathrm{w}, \mathrm{z}\}$ \\
\hline
\end{tabular}

The following sub-sections will introduce the joining rules for the general structure in Fig. 2 by utilizing the ternary Shannon and Davio expansions that were introduced in Section 2.

\subsection{Formal Synthesis of Three-Dimensional ShannON LATtice NeTWORKS}

In general, for $n^{\text {th }}$ radix Galois field logic, no correction functions are needed for lattice networks with $n$-valued Shannon nodes (as will be shown in Theorem 1). This is due to the fact that all of the Shannon cofactors are disjoint (i.e., non-overlapping). So, for instance, for the case of binary Shannon nodes, no correction functions are needed.

Theorem 1. For a lattice network with all ternary Shannon nodes, the following is one possible joining rule:

$J={ }^{0} a J_{0}+{ }^{l} a J_{1}+{ }^{2} a J_{2}$

Proof. By joining in Fig. 2 the following three Shannon nodes in three-dimensional space:

$$
\left[\begin{array}{lll}
1 & 0 & 0 \\
0 & 1 & 0 \\
0 & 0 & 1
\end{array}\right],\left[\begin{array}{lll}
1 & 0 & 0 \\
0 & 1 & 0 \\
0 & 0 & 1
\end{array}\right],\left[\begin{array}{lll}
1 & 0 & 0 \\
0 & 1 & 0 \\
0 & 0 & 1
\end{array}\right]
$$


and by assigning the following 1-RPL values of variable $\{a\}$ for the set of edges $\{\mathrm{r}, \mathrm{s}, \mathrm{t}, \mathrm{u}, \mathrm{v}, \mathrm{w}$, $\mathrm{x}, \mathrm{y}, \mathrm{z}\}$ in Fig. 2:

$$
\begin{aligned}
& t={ }^{0} a, v={ }^{0} a, y={ }^{0} a \\
& r={ }^{l} a, u={ }^{l} a, x={ }^{l} a \\
& s={ }^{2} a, w={ }^{2} a, z={ }^{2} a
\end{aligned}
$$

One obtains the following set of Equations before and after joining the three nodes $\left\{\mathrm{J}_{0}, \mathrm{~J}_{1}, \mathrm{~J}_{2}\right\}$ in Fig. 2, where $\left\{\mathrm{A}, \mathrm{C}, \mathrm{J}_{0}\right\}$ are the set of functions for node $\mathrm{B},\left\{\mathrm{E}, \mathrm{F}, \mathrm{J}_{1}\right\}$ are the set of functions for node $\mathrm{D}$, and $\left\{\mathrm{I}, \mathrm{G}, \mathrm{J}_{2}\right\}$ are the set of functions for node $\mathrm{H}$, respectively:

Before joining the nodes:

$$
\begin{aligned}
& B={ }^{0} a J_{0}+{ }^{l} a C+{ }^{2} a A \\
& D={ }^{0} a E+{ }^{l} a J_{l}+{ }^{2} a F \\
& H={ }^{0} a I+{ }^{l} a G+{ }^{2} a J_{2}
\end{aligned}
$$

After joining the nodes:

$$
\begin{aligned}
B & ={ }^{0} a J+{ }^{1} a C+{ }^{2} a A \\
D & ={ }^{0} a N+{ }^{l} a J+{ }^{2} a F \\
H & ={ }^{0} a I+{ }^{1} a q+{ }^{2} a J
\end{aligned}
$$

Where $N$ and $q$ are the correction functions, and $J$ is the super-imposed node in Fig. 2. By equalizing Equation (20) to Equation (23), Equation (21) to Equation (24), and Equation (22) to Equation (25), and by utilizing the axioms of GF(3), we obtain the following results:

$N=E$

$q=G$

$J={ }^{0} a J_{0}+{ }^{1} a J_{1}+{ }^{2} a J_{2}$

From Equation (19) one observes the fact that the joining rule of any corresponding Shannon decomposition does not need any correction function. The method that has been presented in this sub-section can be used to derive the joining equations for all possible permutations of the elements in the Shannon matrix [S] as well.

\subsection{Formal Synthesis of Three-Dimensional Davio LATTICE NeTWORKS}

In general, for $n^{\text {th }}$ radix Galois logic, at least $(n-1)$ correction functions are needed for lattice circuits with $n$-valued Davio nodes. Thus, for instance, one needs a single correction function in the case of binary Davio expansions, and one needs two correction functions for the case of ternary Davio expansions (as will be shown in the following derivations).

Theorem 2. For a lattice network with all ternary Davio ${ }_{0}\left(D_{0}\right)$ nodes, the following is one possible set of joining rules, and correction functions, respectively:

$$
\begin{aligned}
J & =J_{0} \\
N & =2 a J_{0}+E+a J_{1} \\
q & =2 a J_{0}+G+a J_{2}
\end{aligned}
$$

Proof. By joining in Fig. 2 the following $\mathrm{D}_{0}$ nodes: 
International Journal of VLSI design \& Communication Systems (VLSICS) Vol 11, No 6, December 2020

$$
\left[\begin{array}{lll}
1 & 0 & 0 \\
0 & 2 & 1 \\
2 & 2 & 2
\end{array}\right],\left[\begin{array}{lll}
1 & 0 & 0 \\
0 & 2 & 1 \\
2 & 2 & 2
\end{array}\right],\left[\begin{array}{lll}
1 & 0 & 0 \\
0 & 2 & 1 \\
2 & 2 & 2
\end{array}\right]
$$

and by assigning the following power values of variable $\{a\}$ for the set of edges $\{\mathrm{r}, \mathrm{s}, \mathrm{t}, \mathrm{u}, \mathrm{v}, \mathrm{w}$, $\mathrm{x}, \mathrm{y}, \mathrm{z}$ \} in Fig. 2:

$$
\begin{aligned}
& t=1, v=1, y=1 \\
& r=a, u=a, x=a \\
& s=a^{2}, w=a^{2}, z=a^{2}
\end{aligned}
$$

and by following the same procedure that was used in Theorem 1 (i.e., by using the equivalence of node equations before and after joining the nodes), one obtains:

$$
\begin{aligned}
& J=J_{0} \\
& N=2 a J_{0}+E+a J_{1} \\
& q=2 a J_{0}+G+a J_{2}
\end{aligned}
$$

Theorem 3. For a lattice network with all ternary Davio ${ }_{1}\left(D_{1}\right)$ nodes, the following is one possible set of joining rules, and correction functions, respectively:

$$
\begin{aligned}
& J=J_{0} \\
& N=2 a^{\prime} J_{0}+E+a^{\prime} J_{1} \\
& q=2 a^{\prime} J_{0}+G+a^{\prime} J_{2}
\end{aligned}
$$

Proof. The proof of Theorem 3 follows the same method that is used to prove Theorem 2 .

Theorem 4. For a lattice network with all ternary Davio $2\left(D_{2}\right)$ nodes, the following is one possible set of joining rules, and correction functions, respectively:

$$
\begin{aligned}
& J=J_{0} \\
& N=2 a ” J_{0}+E+a ” J_{1} \\
& q=2 a ” J_{0}+G+a ” J_{2}
\end{aligned}
$$

Proof. The proof of Theorem 4 follows the same method that is used to prove Theorem 2.

The method which is used in Theorems 2 - 4 can be used to derive the joining equations for all possible permutations of the elements in the ternary Davio matrices $\left[\mathrm{D}_{0}\right],\left[\mathrm{D}_{1}\right]$ and $\left[\mathrm{D}_{2}\right][2]$. The structural property of a lattice network depends on the functional property of the decomposed function; if the ternary function is symmetric then there is no need to repeat variables in order to realize the function in three-dimensional lattice network, otherwise there is a need to repeat variables and thus the need to use the results from Theorems $1-4$.

Example 1. For the ternary function $F$ which is represented by the ternary table in Fig. 3, and by utilizing Fig. 2 and the joining operations that were presented in Equations (19) and (29)-(31) for

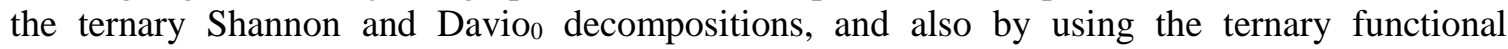
expansions from Section 2, one obtains Figs. 4 and 5 that realize the non-symmetric ternary function $F$ from Fig. 3. As a further detailed explanation, one obtains the three-dimensional lattice network in Fig. 4 by applying the following procedure: 


\begin{tabular}{|c|c|c|}
\hline 0 & 0 & 1 \\
\hline 2 & 1 & 1 \\
\hline 2 & 1 & 1 \\
\hline 2 & 2 & 2 \\
\hline 2
\end{tabular}

Figure 3. Ternary-input ternary-output map for the function: $F=2{ }^{0} \mathrm{a}^{0} \mathrm{~b}+{ }^{0} \mathrm{a}^{1} \mathrm{~b}+{ }^{0} \mathrm{a}^{2} \mathrm{~b}+2{ }^{1} \mathrm{a}^{0} \mathrm{~b}+{ }^{1} \mathrm{a}^{1} \mathrm{~b}+$ $a^{1} b+2{ }^{2} a^{0} b+2{ }^{2} a^{1} b+2{ }^{2} a^{2} b$.

Step1: Expanding nodes.

Expand the non-symmetric function $F=2{ }^{0} \mathrm{a}^{0} \mathrm{~b}+{ }^{0} \mathrm{a}^{1} \mathrm{~b}+{ }^{0} \mathrm{a}^{2} \mathrm{~b}+2{ }^{1} \mathrm{a}^{0} \mathrm{~b}+{ }^{1} \mathrm{a}^{1} \mathrm{~b}+{ }^{1} \mathrm{a}^{2} \mathrm{~b}+2^{2} \mathrm{a}^{0} \mathrm{~b}+$ $2^{2} a^{1} b+2{ }^{2} a^{2} b$ in node $(0,0,0)$ according to Equation (2), as follows:

$\mathrm{F}_{0}=\mathrm{F}(\mathrm{a}=0)=2^{0} \mathrm{~b}+{ }^{1} \mathrm{~b}+{ }^{2} \mathrm{~b}$ into node $(1,0,0)$,

$\mathrm{F}_{1}=\mathrm{F}(\mathrm{a}=1)=2^{0} \mathrm{~b}+{ }^{1} \mathrm{~b}+{ }^{2} \mathrm{~b}$ into node $(0,1,0)$,

$\mathrm{F}_{2}=\mathrm{F}(\mathrm{a}=2)=2^{0} \mathrm{~b}+2^{1} \mathrm{~b}+2^{2} \mathrm{~b}$ into node $(0,0,1)$.

Step 2: Joining nodes.

As a result from step 1 , conflicting values occur in nodes $(1,1,0),(0,1,1)$, and $(1,0,1)$. Join cofactors according to Equation (19), as follows:

$y$-axis cofactor of node $(1,0,0)$ and $x$-axis cofactor of node $(0,1,0)$ into node $(1,1,0) \Rightarrow$ the joined node $(1,1,0)$ is: $1^{1} \mathrm{~b}+2^{0} \mathrm{~b}$,

$z$-axis cofactor of node $(0,1,0)$ and $y$-axis cofactor of node $(0,0,1)$ into node $(0,1,1) \Rightarrow$ the joined node $(0,1,1)$ is: $1^{2} b+2{ }^{1} b$,

$z$-axis cofactor of node $(1,0,0)$ and $x$-axis cofactor of node $(0,0,1)$ into node $(1,0,1) \Rightarrow$ the joined node $(1,0,1)$ is: $1^{2} b+2^{\circ} b$.

Step 3: Expanding nodes.

Expand the lattice nodes that resulted from step 2, as follows:

node $(1,0,0)$ into node $(2,0,0)$ of value 2 ,

node $(1,1,0)$ into nodes: $(2,1,0)$ of value $2,(1,2,0)$ of value 1 , and $(1,1,1)$ of value 0 , node $(0,1,0)$ into node $(0,2,0)$ of value 1 ,

node $(0,1,1)$ into nodes: $(0,2,1)$ of value $2,(0,1,2)$ of value 1 , and $(1,1,1)$ of value 0 , node $(0,0,1)$ into node $(0,0,2)$ of value 2 ,

node $(1,0,1)$ into nodes: $(2,0,1)$ of value $2,(1,0,2)$ of value 1 , and $(1,1,1)$ of value 0 .

By applying the same previous procedure of expanding-joining steps, and by utilizing the expansion in Equation (16) for expansion nodes of type $D_{0}=\left[\begin{array}{lll}1 & 0 & 0 \\ 0 & 2 & 1 \\ 2 & 2 & 2\end{array}\right]$, and the joining operations in Theorem 2, one obtains the three-dimensional network which is presented in Fig. 5. Note that, by joining cofactors according to Equations (29) - (31), the repetition of variable $\{b\}$ is performed, and therefore a new level in the three-dimensional lattice network is obtained in order to create the corresponding leaves with non-conflicting values. 


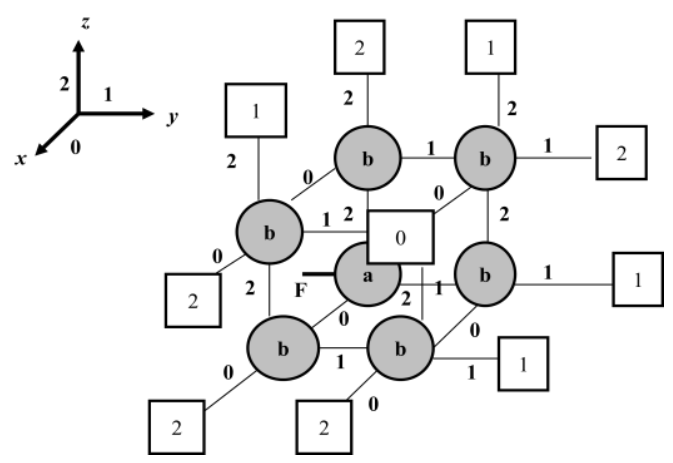

Figure 4. Three-dimensional Shannon lattice network for the realization of the non-symmetric function in Fig. 3: $F=2^{0} a^{0} b+{ }^{0} a^{1} b+2{ }^{1} a^{0} b+{ }^{1} a^{1} b+{ }^{1} a^{2} b+2{ }^{2} a^{2} b+2{ }^{2} a^{1} b+2{ }^{2} a^{0} b+{ }^{0} a^{2} b$.

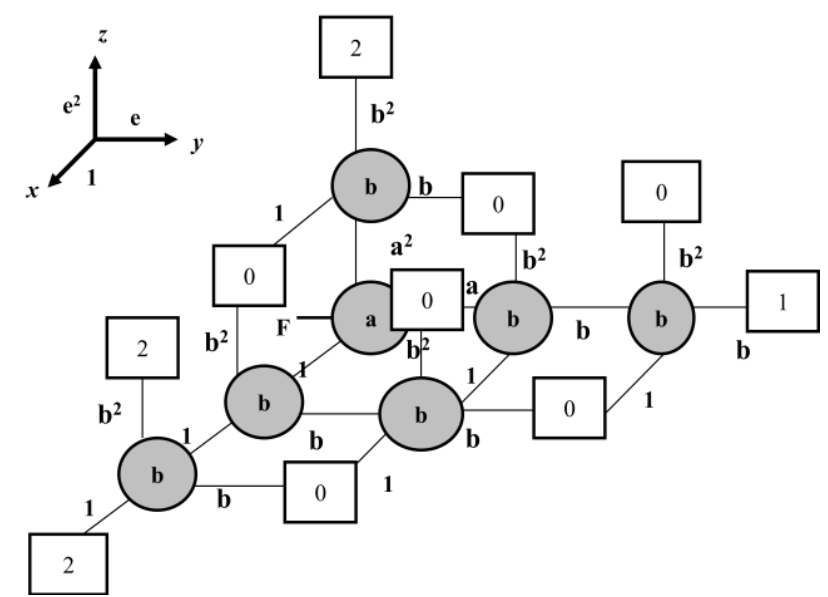

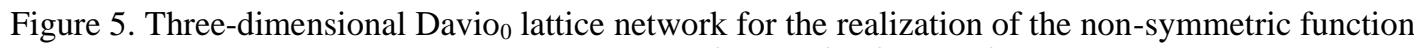
in Fig. 3: $F=2+a \cdot b^{2}+2 \cdot a^{2} \cdot b^{2}+2 \cdot b^{2}$.

By observing Figs. 4 and 5, one obtains the following size-based comparison shown in Table 3.

Table 3. Size-based comparison between the three-dimensional lattice realizations in Figs. 4 and 5 for the non-symmetric function $F=2{ }^{0} a^{0} b+{ }^{0} a^{1} b+{ }^{0} a^{2} b+2{ }^{1} a^{0} b+{ }^{1} a^{1} b+{ }^{1} a^{2} b+2{ }^{2} a^{0} b+2{ }^{2} a^{1} b+2{ }^{2} a^{2} b$.

\begin{tabular}{lcc}
\hline Parameter & 3D Shannon Lattice (Fig. 4) & 3D Davio \\
\hline Total \# of Intice (Fig. 5) \\
Total \# of Leaves & 7 & 7 \\
Total \# of Zero-Valued Leaves & 10 & 10 \\
\hline
\end{tabular}

One can note, for example, that while the Shannon lattice network in Fig. 4 has only one zero-

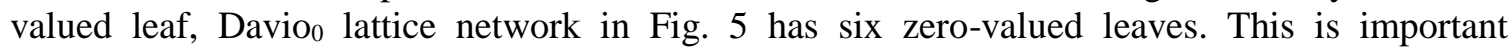
especially in hardware implementation when considering power consumption in such lattice realizations, since value " 0 " represents ground and thus does not need to be supplied from a power supply, in contrast to values " 1 " and " 2 " that are obtained from a power supply and thus consume more power in total. Also, as an initial evaluation, Example 1 shows the importance of performing the correct selection of internal node type (e.g., Shannon nodes in Fig. 4 and Davio $_{0}$ nodes in Fig. 5) in order to obtain optimal results of the criteria for which one is designing, such as power consumption, performance (i.e., delay) or size. 


\section{Multi-Valued Processing Using Carbon Field Emission - Based CONTROLLED SWITCHING}

This section presents carbon field emission - based controlled switching, and the corresponding utilization within multi-valued Galois computing [9]. The implementation of controlled switching that use carbon field emission - based devices within the corresponding synthesis of threedimensional lattice networks (which will be introduced in Section 5) will utilize the accumulative results which are presented in the following three sub-sections.

\subsection{Two-To-One Controlled SwITCHING}

By the utilization of the previously experimented and observed characterizations and operations of carbon field-emission from the corresponding nano-apex carbon fibers and CNTs that were presented in the first part of this article, Fig. 6 presents the carbon field emission - based primitive that realizes the two-to-one controlled switching. In Fig. 6, the input control signal that is used to control the electric conduct of the device is implemented using the imposed electric field intensity $(E)$ or equivalently the work function $(\Phi)$ or voltage $(V)$.

The description of the operation of the carbon field emission - based device shown in Fig. 6(b) is as follows: by imposing the control signal of high voltage (HV), the voltage difference between the carbon cathodes and the facing anode is varied. This change will make the carbon cathode with control signal (HV) to be field emitting while the other carbon cathode with the complementary control signal $(\overline{\mathrm{HV}})$ to be without field emission. When the voltage difference is reversed, the carbon cathode with the complementary control signal $(\overline{\mathrm{HV}})$ will be field emitting while the other carbon cathode with the control signal (HV) will be without field emission. Thus, this device implements the functionality of the 2-to-1 controlled switching $(G=a c+b c$ ') which is shown in Fig. 6(a).

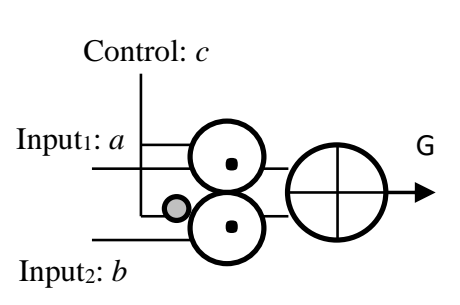

(a)

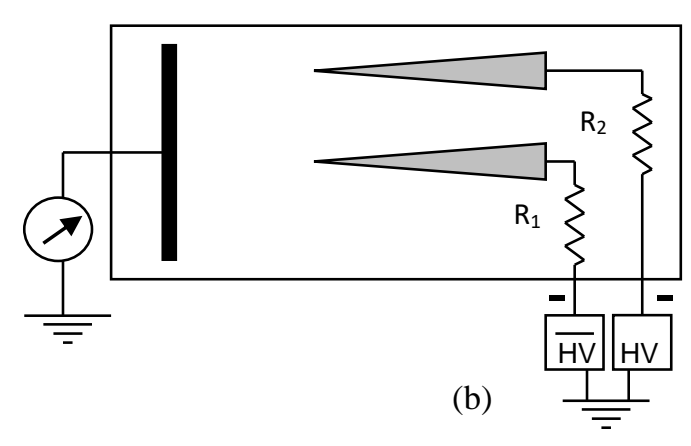

(b)

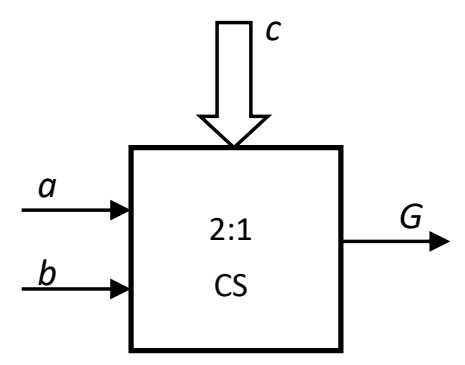

(c)

Figure 6. The carbon field emission - based device implementing the operation of the two-to-one controlled switching (CS): (a) two-to-one multiplexer ( $G=a c+b \bar{c})$, (b) the carbon field emission-based two-to-one CS, and (c) block diagram for the new two-to-one multiplexer. 
The experimental results show that the distance $d$ which is required between the cathodes and the facing anode must be generally around $10 \mathrm{~mm}$, else beam distortion will occur and will be affecting the collected current at the facing anode screen. Since the equations that relate the electric field intensity $(E)$, work (i.e., energy) function $(\Phi)$ in Joules $(\mathrm{J})$, distance $(d)$, voltage $(V)$, and the current density $(J)$, are as follows:

$$
\begin{aligned}
& \Phi=e \cdot V \\
& V=E \cdot d \\
& d=V / E \\
& J=I /(a / \Omega)=I \cdot(\Omega / a)
\end{aligned}
$$

where $e$ is the electron charge $\cong 1.602 \cdot 10^{-19} \mathrm{C}, a$ is the tip area and $\Omega$ is the emission angle, then the equation that models the current value on the anode screen can be derived as follows:

$$
I=\left(\frac{a A}{\Omega d^{2}} e^{-\frac{B d}{V}}\right) V^{2}
$$

From Equation (45), one can clearly observe the corresponding proportionality relation between the current value $I$ and the voltage difference $V$, proportionality relation between the current value $I$ and the tip area $a$, and the inverse relation between the current value $I$ and the emission angle $\Omega$, where $A=1.541 \times 10^{-6} / \Phi$ and $B=6.831 \times 10^{9} \Phi^{3 / 2}$. For example, the value of the work function is $\Phi=4.5 \mathrm{eV}$ for tungsten and can be set to $\Phi=4.9 \mathrm{eV}$ for graphene, where the typical value used for the experiment input control variable of the electric field intensity $E$ is $\geq 3 \cdot 10^{9}$ $\mathrm{V} / \mathrm{m}$, for the distance $d$ between the CNT cathode and the facing anode screen is $\cong 10 \mathrm{~mm}$, and for the applied voltage $V \cong 3 \cdot 10^{7} \mathrm{~V}$.

\subsection{The Extension to Many-To-One Controlled Switching}

Synthesizing many-to-one carbon field emission - based controlled switching is possible using the fundamental two-to-one carbon field emission - based controlled switch from Fig. 6(b). For example, for the three-valued logic case, one needs two devices $\left\{D_{1}, D_{2}\right\}$ from Fig. $6(b)$ to realize the functionality of three-to-one carbon field emission - based controlled switching. This idea is illustrated in Fig. 7.

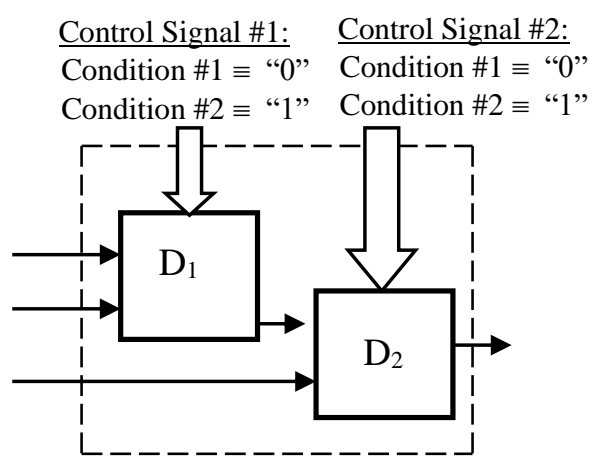

Figure 7. The realized carbon-based three-to-one field-emission controlled switching. 
Note that, in Fig. 7, device $\mathrm{D}_{1}$ outputs one signal from two input signals and device $\mathrm{D}_{2}$ outputs one signal from two input signals, thus the total functionality of the device in Fig. 7 is a three-toone carbon field emission - based controlled switching. In general, for the case of $m$-valued logic, one needs $(m-1)$ of the two-to-one controlled switches to realize the function of an $m$-to-1 controlled switching. This idea is illustrated in Fig. 8.

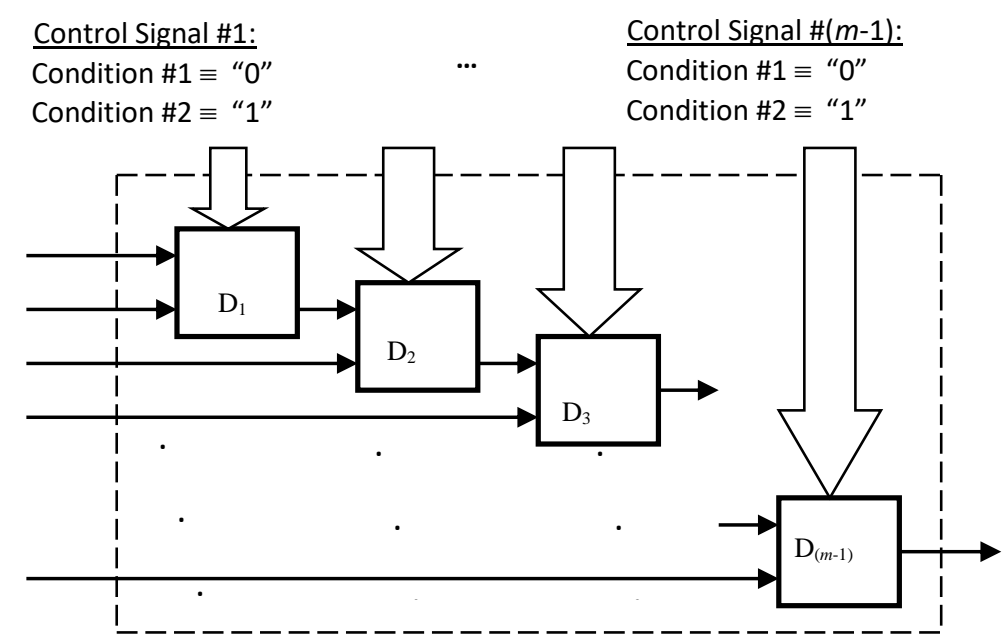

Figure 8 . The realization of an (m-to-1) controlled switching, where devices $\left\{\mathrm{D}_{1}, \ldots, \mathrm{D}_{(\mathrm{m}-1)}\right\}$ can be the carbon-based field emission controlled switch from Fig. 6(b).

\subsection{Multi-Valued Computing Using Carbon Field Emission-BaSed DeviceS}

Multi-valued computing will be illustrated in this sub-section using the carbon-based field emission device that was previously developed for the case of GF(3). A controlled switch - based circuit that implements GF(3) addition and multiplication tables is shown in Fig. 9, where Fig. 9(a) can be implemented using the two-input single-output carbon field-emission device that was shown in Fig. 6(b).

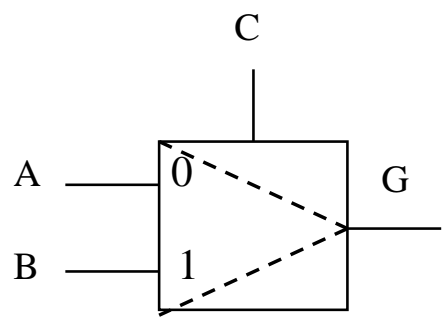

\begin{tabular}{|l|l|l||l|}
\hline $\mathrm{A}$ & $\mathrm{B}$ & $\mathrm{C}$ & $\mathrm{G}$ \\
\hline $\mathrm{a}$ & $\mathrm{b}$ & 0 & $\mathrm{a}$ \\
\hline $\mathrm{a}$ & $\mathrm{b}$ & 1 & $\mathrm{~b}$ \\
\hline
\end{tabular}

(a)

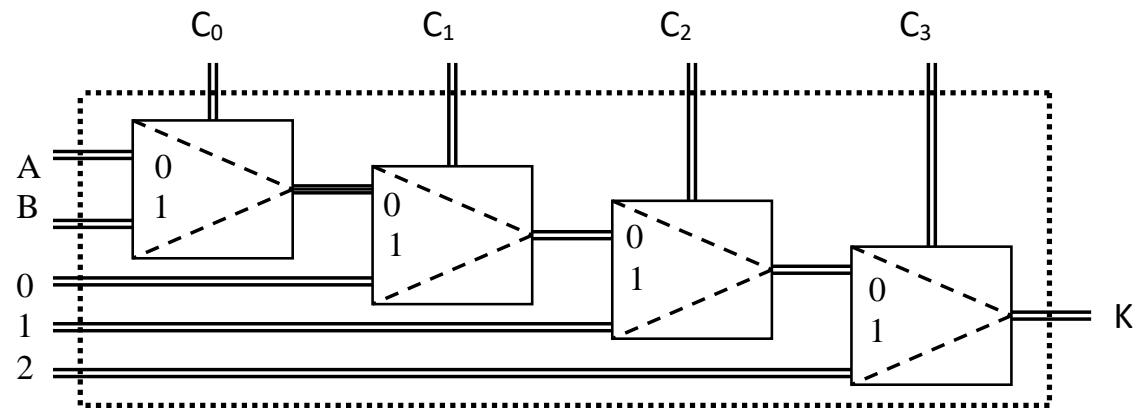

(b)

Figure 9. Galois arithmetic implementation using carbon - based switching: (a) symbol of controlledswitching that can be realized using the device in Fig. 6(b), and (b) circuit that uses controlled-switching to implement the corresponding GF(3) addition and multiplication operations. 
In Fig. 9(b), variables $\{A, B\}$ are two ternary input variables that can take any value from the set $\{0,1,2\}$, inputs $\{0,1,2\}$ are constant inputs, and inputs $C_{k}(k=0,1,2,3)$ are two-valued control variables that take values from the set $\{0,1\}$. Note that Fig. $9(\mathrm{~b})$ implements $\mathrm{GF}(3)$ addition and multiplication tables by using the appropriate values of control variables $C_{k}$ that select the variable inputs $\{A, B\}$ and constant inputs $\{0,1,2\}$. For instance, Table 4 shows an example for the implementation of $\mathrm{GF}(3)$ addition and multiplication tables using Fig. 9(b).

Table 4. An example for the implementation of GF(3) addition and multiplication tables using Fig. 9(b), where + means GF(3) addition, * means GF(3) multiplication, $C_{k}(+)$ means that the control variable $C_{k}$ to implement the ternary addition operation, and $C_{k}(*)$ means that the control variable $C_{k}$ to implement the ternary multiplication operation.

\begin{tabular}{|l|l||l|l|l|l||l|l|l|l||l|l|}
\hline $\mathbf{A}$ & $\mathbf{B}$ & $\mathbf{C}_{\mathbf{0}}(+)$ & $\mathbf{C}_{\mathbf{1}}(+)$ & $\mathbf{C}_{\mathbf{2}}(+)$ & $\mathbf{C}_{\mathbf{3}}(+)$ & $\mathbf{C}_{\mathbf{0}}(*)$ & $\mathbf{C}_{\mathbf{1}}(\boldsymbol{*})$ & $\mathbf{C}_{\mathbf{2}}(*)$ & $\mathbf{C}_{\mathbf{3}}(*)$ & + & $*$ \\
\hline \hline 0 & 0 & 0 & 0 & 0 & 0 & 0 & 0 & 0 & 0 & 0 & 0 \\
\hline 0 & 1 & 1 & 0 & 0 & 0 & 0 & 0 & 0 & 0 & 1 & 0 \\
\hline 0 & 2 & 1 & 0 & 0 & 0 & 0 & 0 & 0 & 0 & 2 & 0 \\
\hline 1 & 0 & 0 & 0 & 0 & 0 & 1 & 0 & 0 & 0 & 1 & 0 \\
\hline 1 & 1 & 0 & 0 & 0 & 1 & 0 & 0 & 0 & 0 & 2 & 1 \\
\hline 1 & 2 & 0 & 1 & 0 & 0 & 1 & 0 & 0 & 0 & 0 & 2 \\
\hline 2 & 0 & 0 & 0 & 0 & 0 & 1 & 0 & 0 & 0 & 2 & 0 \\
\hline 2 & 1 & 0 & 1 & 0 & 0 & 0 & 0 & 0 & 0 & 0 & 2 \\
\hline 2 & 2 & 0 & 0 & 1 & 0 & 0 & 0 & 1 & 0 & 1 & 1 \\
\hline
\end{tabular}

For the internal nano interconnects in Fig. 9(b), they can be implemented using CNTs as shown in Fig. 10, where the symbol = means a metallic CNT used as a nanowire, especially as the CNT possesses the important properties of small size, high resilience and very low electron scattering as was shown in the first part of the article. Several efficient methods for implementing such interconnects have been reported by growing a SWCNT between two metal catalyst islands such as iron $(\mathrm{Fe})$, cobalt $(\mathrm{Co})$, nickel $(\mathrm{Ni})$, yttrium $(\mathrm{Y})$ or molybdenum $(\mathrm{Mo})$.

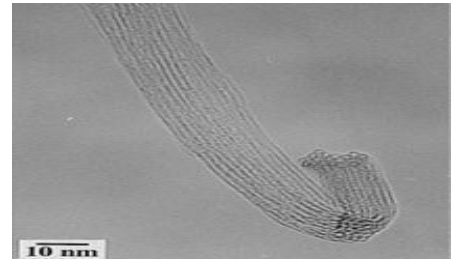

(a)

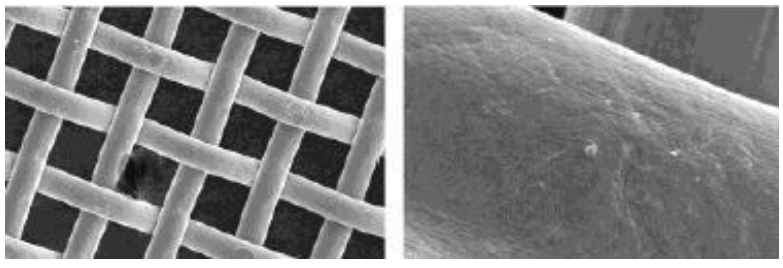

(b)

Figure 10. The implementation of CNT-based nano interconnects within controlled-switching: (a) Transmission Electron Microscopy (TEM) image of a bundle of SWCNTs catalyzed by Ni/Y mixture, and (b) growth of CNT wires on catalysts where CNT meshes are shown on which the metal catalyst is coated.

Since multi-valued circuits over GF(3) will be synthesized using the addition and multiplication operations, the circuit which is shown in Fig. 9(b) can be used in multi-valued implementations whenever the corresponding GF(3) addition and multiplication operations are applied, and the internal nano interconnects can be implemented using the corresponding metallic CNTs. The following Example 2 demonstrates the system-level design of an Arithmetic and Logic Unit (ALU) by illustrating the implementation of a 2-digit multiplier using the introduced carbonbased field-emission switching devices. 
Example 2. A 2-digit multi-valued multiplication is performed utilizing the mod-multiplication operator. Table 5 shows the general maps for the ternary multiplication and carry out $\left(C_{\text {out }}\right)$ functions, and Fig. 11 shows the corresponding three-valued circuit realization.

Table 5. Ternary multiplication tables: (a) ternary multiplication function, and (b) ternary carry out function for the ternary two-digit multiplier.

(a)

\begin{tabular}{|l|l|l|l|}
\hline $\mathbf{x}$ & $\mathbf{0}$ & $\mathbf{1}$ & $\mathbf{2}$ \\
\hline $\mathbf{0}$ & 0 & 0 & 0 \\
\hline $\mathbf{1}$ & 0 & 1 & 2 \\
\hline $\mathbf{2}$ & 0 & 2 & 1 \\
\hline
\end{tabular}

(b)

\begin{tabular}{|l|l|l|l|}
\hline $\mathbf{x}$ & $\mathbf{0}$ & $\mathbf{1}$ & $\mathbf{2}$ \\
\hline $\mathbf{0}$ & 0 & 0 & 0 \\
\hline $\mathbf{1}$ & 0 & 0 & 0 \\
\hline $\mathbf{2}$ & 0 & 0 & 1 \\
\hline
\end{tabular}

The corresponding GF(3) addition and multiplication operations that are used in Fig. 11 could be implemented using the results which were demonstrated in Table 4 that specifies input values within Fig. 9(b) to the various inputs.

Further implementation of the general multi-valued $N$-bit full ALU, which is the main functional unit in the data path within the microprocessor, that includes the realization of all arithmetic subunits of \{addition, subtraction, multiplication, division \} and all logic sub-units of \{NOT, AND, OR, XOR \} can be performed from the utilization of the carbon-based controlled switching device (which was previously introduced) by using the same method which is used in the realization of the 2-digit multiplier that is shown in Fig. 11.

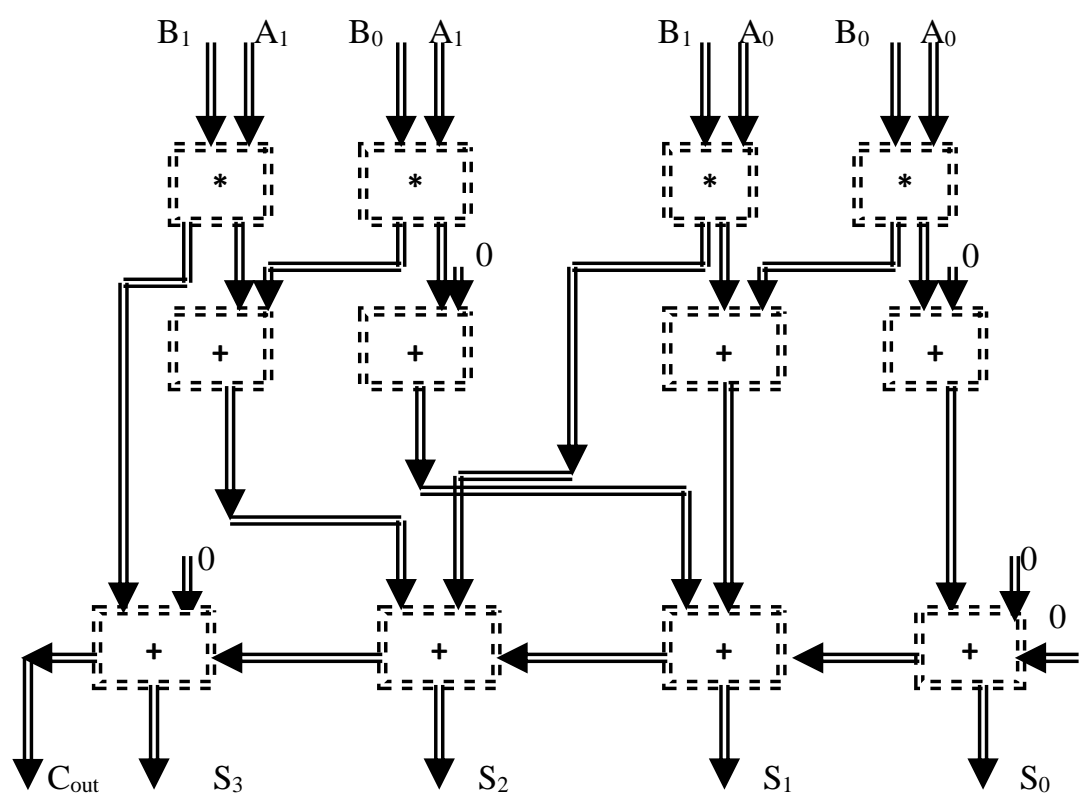

Figure 11. Logic circuit of a ternary two-digit multiplier. 


\section{Concurrent Three-Dimensional Lattice Processing Via CARBON - EMISSION SWITCHING}

This section introduces the synthesis of three-valued Galois functions using carbon field emission - based three-dimensional lattice networks utilizing the method shown in Fig. 12. In this method, mapping three-valued functions into the corresponding three-dimensional lattice network can be achieved using either the form of function expression obtained directly through the RPL-based decomposition, or by using the function tabular form of the corresponding three-valued function.

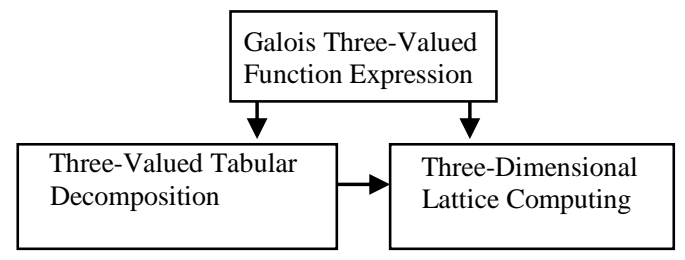

Figure 12. Utilized method to realize three-valued Galois logic by using function decompositions.

Example 3 shows the realization of a ternary non-symmetric function in a three-dimensional lattice network through the repetition of variables and utilizing the synthesis scheme from Fig. 12 , where the operations performed in each nano node in the corresponding three-dimensional lattice network can be implemented using the nano circuit from Fig. 9(b) and the corresponding specified input values from Table 4.

Example 3. For the non-symmetric two-variable three-valued function $F=a b+a^{\prime} b^{\prime \prime}$ shown in Fig. 13, and by adopting the right-hand rule of the Cartesian coordinate system, Fig. 14 illustrates the three-dimensional lattice network implementation for such non-symmetric function. In Fig. 14 , if one multiplies each leaf value, going counter clock wise, with all possible out-to-in paths (i.e., from the leaves to the root) and adds them over Galois field then one obtains the corresponding map, where $\left\{{ }^{0} a,{ }^{1} a,{ }^{2} a\right\}$ are the zero, first and second polarities of the 1-RPL of variable $a,\left\{{ }^{0} b,{ }^{1} b,{ }^{2} b\right\}$ are the zero, first and second polarities of the 1-RPL of variable $b$, and variables $a$ and $b$ can take any value in the set $\{0,1,2\}$.

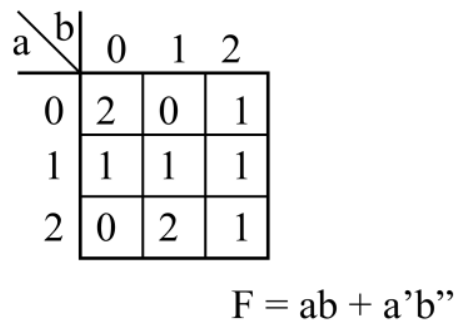

(a)

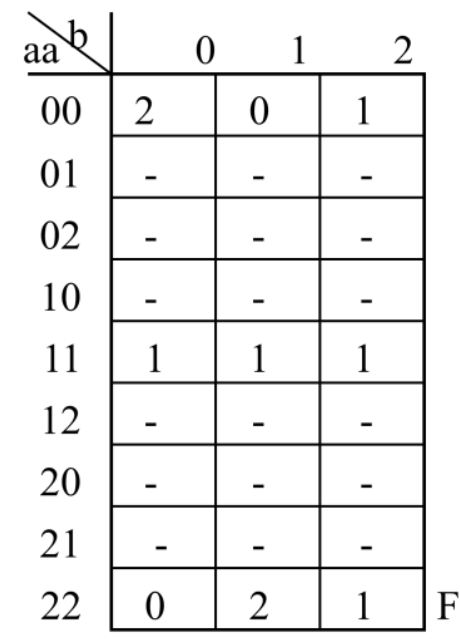

(b)

Figure 13. The process of symmetrization of ternary functions: (a) Three-valued function which is nonsymmetric, and (b) repeating variable $\{a\}$ to achieve symmetrization, where $\left\{a^{\prime}\right\}$ is a single shift to the value of the variable $\{a\}$ and $\left\{b^{\prime \prime}\right\}$ is double shifts to the value of variable $\{b\}$. 


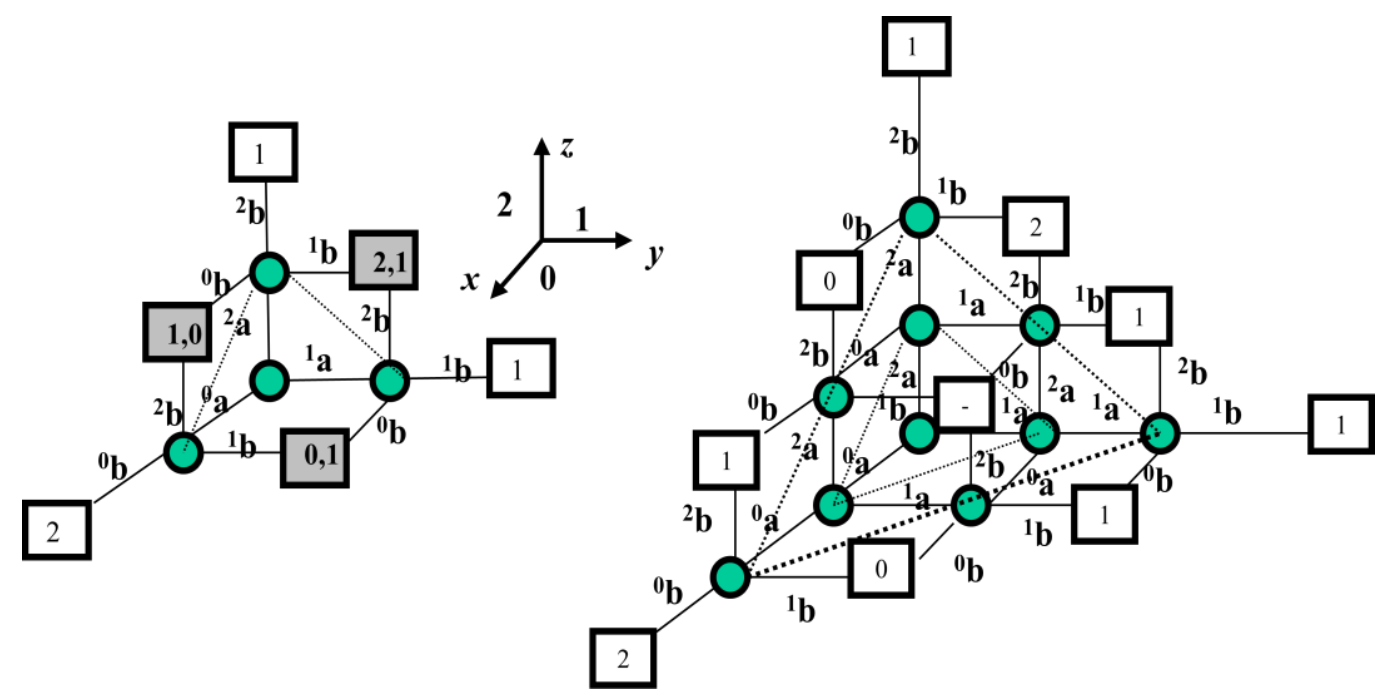

(a)

(b)

Figure 14. Regular nano-based three-dimensional lattice networks: (a) three-dimensional lattice network that corresponds to Fig. 13(a) with conflicting leaves (in dark boxes), and (b) the final three-dimensional lattice network that corresponds to Fig. 13(b) with non-conflicting leaves.

The resulting synthesized three-dimensional lattice networks (such as in Fig. 14) have the characteristics of the full utilization of high regularity and thus compactness in three-dimensional space, the relative ease of manufacturability and testability, and the lower power consumption which is due to the use of only local interconnects and low-power carbon-based nano switches, where (as mentioned previously and in general for the corresponding three-valued Shannon and Davio lattice networks) the operations performed in each internal node in the resulting threedimensional lattice networks can be implemented using the nano switching circuit from Fig. 9(b) and by utilizing the corresponding specified input values from Table 4.

\section{Conclusions}

The formalistic design of regular three-dimensional lattice networks for the novel realization of three-valued Galois functions using carbon field emission - based nano switching devices is introduced. The generalized implementation of lattice networks using the corresponding many-toone carbon-based field emission controlled-switching devices is also presented. This is performed through the realization of multi-valued processing by implementing the corresponding Galois arithmetic operations that utilize the serial interconnects of several two-to-one basic controlledswitching elements, where each of these basic elements can be directly implemented using the presented carbon-based field emission devices.

Novel realizations of the operations for three-valued lattice systems utilizing the introduced nanobased architectures are presented, within which highly-regular three-dimensional lattice networks generalize the concept of two-dimensional four-neighbour lattices into three-dimensional sixneighbour lattice networks. In general, lattice networks possess the important property of high regularity, which is useful in several implementations such as within fault testing and localization, self-repair, compactness and ease of manufacturability. 
Other advantages of the highly-regular three-dimensional lattice networks include reasons such as no need for three-dimensional routing and placement analogously to the two-dimensional case. Further, and because of using only local interconnects and the utilization of efficient carbonbased nano switching, the presented lattice networks can be utilized within three-dimensional technologies for which minimal power consumption is required.

The presented field-emission switching architectures in this part will be further utilized in the third part of the article within the architectural synthesis of controlled-switching that will be used in the layout congestion-free design of concurrent nano-based lattice networks and systems.

\section{ACKNOWLEDGEMENT}

This research was performed during sabbatical leave in 2019-2020 granted to the author from The University of Jordan and spent at Isra University, Jordan.

\section{REFERENCES}

[1] S. B. Akers, "A rectangular logic array," IEEE Transactions on Computers, Vol. C-21, pp. 848-857, 1972.

[2] A. N. Al-Rabadi, Reversible Logic Synthesis: From Fundamentals to Quantum Computing, SpringerVerlag, New York, 2004.

[3] A. N. Al-Rabadi, "Three-dimensional lattice logic circuits, part II: formal methods," Facta Universitatis $(F U)$ - Electronics and Energetics, Vol. 18, No. 1, pp. 15 - 28, 2005.

[4] A. N. Al-Rabadi and M. Perkowski, "New families of reversible expansions and their regular lattice circuits," Journal of Multiple-Valued Logic and Soft Computing (MVLSC), Old City Publishing, U.S.A., Vol. 11, No. 3-4, pp. 213 - 238, 2005.

[5] A. N. Al-Rabadi, "New dimensions in non-classical neural computing, Part I: three-dimensionality, invertibility, and reversibility," International Journal of Intelligent Computing and Cybernetics, Emerald, Vol. 2, No. 2, pp. 348 - 385, 2009.

[6] A. N. Al-Rabadi, "New dimensions in non-classical neural computing, Part II: quantum, nano, and optical," International Journal of Intelligent Computing and Cybernetics, Emerald, Vol. 2, No. 3, pp. 513-573, 2009.

[7] A. N. Al-Rabadi, Carbon NanoTube (CNT) Multiplexers, Circuits, and Actuators, United States Patent and Trademark Office, Patent No. US 7,508,039 B2, U.S.A., 2009.

[8] A. N. Al-Rabadi, "Circuits for $m$-valued classical, reversible and quantum optical computing with application to regular logic design," Int. Journal of Intelligent Computing and Cybernetics (IJICC), Emerald, Vol. 2, No. 1, pp. 52 - 101, 2009.

[9] A. N. Al-Rabadi, M. S. Mousa, R. F. Al-Rabadi, S. Alnawasreh, R. F. Altrabsheh, and M. A. Madanat, "Parallel bijective processing of regular nano systolic grids via carbon field emission controlled-switching," Int. Journal of Computer Science \& Information Technology (IJCSIT), AIRCC Publishing Corporation, Vol. 8, No. 3, pp. 77-101, 2016.

[10] S. Alnawasreh, M. S. Mousa, and A. N. Al-Rabadi, "Investigating the effects of sample conditioning on nano-apex carbon fiber tips for efficient field electron emission," Jordan J. Physics, Vol. 8, No. 1, pp. 51-57, 2015.

[11] K. Alonso and M. J. Hagmann, "Comparison of three different methods for coupling of microwave and terahertz signals generated by resonant laser-assisted field emission," Journal of Vacuum Science \& Technology B: Microelectronics and Nanometer Structures, Vol. 19, No. 1, pp. 68-71, 2001.

[12] G. Amaratunga, "Watching the nanotube," IEEE Spectrum, pp. 28-32, 2003.

[13] J. M. Bonard, K. A. Dean, B. F. Coll, and C. Klinke, "Field emission of individual carbon nanotubes in the scanning electron microscope," Physical Review Letters, 89(19):197602-1:4, 2002.

[14] J. M. Bonard, J. P. Salvetat, T. Stöckli, L. Forro, and A. Chatelain, "Field emission from carbon nanotubes: perspectives for applications and clues to the emission mechanism," Applied Physics A: Materials Science \& Processing, Vol. 69, No. 3, pp. 245-254, 1999. 
[15] M. Brugat, M. S. Mousa, E. P. Sheshin, and M. J. Hagmann, "Measurement of field emission current variations caused by an amplitude modulated laser," Materials Science and Engineering A, 327(1):715, 2002.

[16] R. Bryant, "Graph-based algorithms for Boolean functions manipulation," IEEE Trans. on Comp., Vol. C-35, No. 8, pp. 667-691, 1986.

[17] P. J. Burke, "Luttinger liquid theory as a model of the gigahertz electrical properties of carbon nanotubes," IEEE Transactions on Nanotechnology, 1(3):129-144, 2002.

[18] H. F. Cheng, Y. S. Hsieh, Y. C. Chen, and I. N. Lin, "Laser irradiation effect on electron field emission properties of carbon nanotubes," Diamond and Related Materials, 13(4-8):1004-1007, 2004.

[19] A. N. Cleland, Foundations of Nanomechanics: From Solid State Theory to Device Applications, Springer-Verlag, 2003.

[20] P. G. Collins and P. Avouris, "Nanotubes for electronics," Scientific American, pp. 62-69, 2000.

[21] P. G. Collins, M. S. Arnold, and P. Avouris, "Engineering carbon nanotubes and nanotube circuits using electrical breakdown," Science, Vol. 292, 2001.

[22] V. Derycke, R. Martel, J. Appenzeller, and P. Avouris, "Carbon nanotube inter- and intramolecular logic gates," Nano Letters, Vol. 0, No. 0, A - D, 2001.

[23] E. Drexler, Engines of Creation: the Coming Era of Nanotechnology, Anchor Books, 1986.

[24] R. G. Forbes, "Extraction of emission parameters for large-area field emitters, using a technically complete Fowler-Nordheim-type equation," Nanotechnology, IOP Publishing, 23(9), 2012.

[25] R. H. Fowler and L. W. Nordheim, "Electron emission in intense electric fields," Proceedings of the Royal Society A:Mathematical, Physical and Engineering Sciences, 119(781):137-181, 1928.

[26] T. Fujieda, K. Hidaka, M. Hayashibara, T. Kamino, Y. Ose, H. Abe, T. Shimizu, and H. Tokumoto, "Direct observation of field emission sites in a single multiwalled carbon nanotube by Lorenz microscopy," Japanese J. Applied Physics, 44(4A):1661-1664, 2005.

[27] D. H. Green, "Families of Reed-Muller canonical forms," International Journal of Electronics, No. 2, pp. 259-280, 1991.

[28] P. Hommelhoff, Y. Sortais, A. Aghajani-Talesh, and M. A. Kasevich, "Field emission tip as a nanometer source of free electron femtosecond pulses," Physical Review Letters, 96(7):077401-1:4, 2006.

[29] J. Jiao, E. Einarsson, D. W. Tuggle, L. Love, J. Prado, and G. M. Coia, "High-yield synthesis of carbon coils on tungsten substrates and their behavior in the presence of an electric field," $J$. Materials Research, Vol. 18, No. 11, pp. 2580-2587, 2003.

[30] M. G. Karpovsky, Finite Orthogonal Series in the Design of Digital Devices, Wiley, New York, 1976.

[31] Z. Kohavi, Switching and Finite Automata Theory, McGraw-Hill, New York, 1978.

[32] K. Likharev, "Electronics below 10 nm," in: Nano and Giga Challenges in Microelectronics, pp. 2768, Elsevier, 2003.

[33] M. M. Mano and C. R. Kime, Logic and Computer Design Fundamentals, $4^{\text {th }}$ edition, Prentice-Hall, 2008.

[34] A. N. Obraztsov, I. Pavlovsky, A. P. Volkov, E. D. Obraztsova, A. L. Chuvilin, and V. L. Kuznetsov, "Aligned carbon nanotube films for cold cathode applications," Journal of Vacuum Science \& Technology B, 18(2): 1059-1063, 2000.

[35] K. C. Smith, "Prospects for VLSI technologies in MVL," Booklet of Ultra Large Scale Integration (ULSI) Workshop, p.4, Boston, Massachusetts, 2002.

[36] R. S. Stankovic, Spectral Transform Decision Diagrams in Simple Questions and Simple Answers, Nauka, Belgrade, 1998.

[37] R. S. Stankovic, J. Astola, and C. Moraga, "Fast Fourier transforms on finite groups as a method in synthesis for regularity," Journal of Multiple-Valued Logic and Soft Computing, 23(5-6), pp. 463483, 2014.

[38] D. Tomanek, R. Enbody, K. Young-Kyun, and M. W. Brehob, "Nanocapsules containing charged particles, their uses and methods of forming the same," U.S. Patent, Publication No.: US 2002/0027819 A1, 2002.

[39] T. J. Webster, M. C. Waid, J. L. McKenzie, R. L. Price, and J. U. Ejiofor, "Nano-biotechnology: carbon nanofibres as improved neural and orthopaedic implants," Nanotechnology, 15, pp. 48-54, 2004. 
[40] T. Zhang, J. Chang, W. Su, X.-L. Zhou, and X. Jia, "Thermoelectric properties of antimony films: roles of oxidation and topological quantum state," Nanotechnology, 31(48), 2020.

[41] L. Zhang, S. Fahad, H.-R. Wu, T.-T. Dong, Z.-Z. Chen, Z.-Q. Zhang, R.-T. Liu, X.-P. Zhai, X.-Y. Li, X. Fei, Q.-W. Song, Z.-J. Wang, L.-C. Chen, C.-L. Sun, Y. Peng, Q. Wang, and H.-L. Zhang, "Tunable nonlinear optical responses andcarrier dynamics of two-dimensional antimonene nanosheets," Nanoscale Horizons, 5(10), pp. 1420-1429, 2020.

\section{AUTHOR}

Anas N. Al-Rabadi is currently a Professor in the Computer Engineering Department at The University of Jordan. He received his Ph.D. in Computer Design and Advanced Logic Synthesis in 2002 and M.Sc. in Control and Power Electronic Systems Design in 1998, both from the Department of Electrical and Computer Engineering at Portland State University. Prof. Al-Rabadi had served as a Research Faculty at the Office of Graduate Studies and Research (OGSR) at Portland State University from 2002 until 2004. He is the author of the first comprehensive book and the first international published title on Reversible Logic Synthesis, Reversible Logic Synthesis: From Fundamentals to Quantum Computing (Springer-Verlag, 2004). Currently, Prof. Al-Rabadi is the author of more than 130 international indexed and scholarly publications, in addition to a registered U.S.A. nanotechnology patent in 2009. His current research includes distributed and parallel computing, systolic architectures, regular circuits and systems, reversible logic, quantum computing, multiple-valued logic, machine learning, artificial intelligence, soft computing and computational intelligence, optical computing, reconstructability analysis of systems, signal processing, spectral methods, testing and design for testability, nanotechnology, robotics, optimal and robust control, and digital error-control coding. 